\title{
Fabrication and characterization of phosphorus-doped diamond field emitters in triode-type field emission arrays
}

\author{
Chia-Fu Chen*, Chia-Lun Tsai, Chien-Liang Lin \\ Department of Materials Science and Engineering, National Chiao Tung University, 1001 Ta Hsueh Road, Hsinchu 30050, Taiwan, ROC
}

\begin{abstract}
In this work, we present a novel scheme that involves a new fabrication process for gate-structured metal-insulator-semiconductor (MIS) diodes using IC technology. Using a bias-assisted microwave-plasma chemical vapor deposition (BAMPCVD) system to synthesize P-doped emitters completes this process. A comparison of the field emission characteristics of two types of non-doped dendrite-like and P-doped nanotube-like diamond emitters with a 4- $\mu \mathrm{m}$ gate aperture is made. Phosphorus doping can enhance the electrical characteristics by reducing the turn-on voltage and enhancing the emission current density. The turn-on voltage of non-doped and P-doped emitters is 15 and $5 \mathrm{~V}$, respectively. Phosphorus doping can enhance the electrical properties by increasing the emission current, since the field emission current $\left(I_{\mathrm{a}}\right)$ of non-doped and P-doped emitters is $4 \mu \mathrm{A}$ (at $V_{\mathrm{gc}}=45 \mathrm{~V}$ ) and $322 \mu \mathrm{A}$ (at $V_{\mathrm{gc}}=120 \mathrm{~V}$ ), respectively. The emission current of P-doped emitters is approximately 80-fold larger than that of non-doped diamond emitters. (c) 2001 Elsevier Science B.V. All rights reserved.
\end{abstract}

Keywords: Field emission; Diamond; Chemical vapor deposition (CVD); n-Type doping

\section{Introduction}

Field emission display (FED) is evolving as one of the promising techniques for future generations of flat panel displays (FPD). Indeed, there have been several efforts to fabricate metal-tip emitters and silicon-tip array field-emission devices. However, the electrical field required for triggering the field emission of these devices is rather high. Moreover, their performance rapidly degrades, due to the thermal effect [1].

In contrast, diamond has recently been considered as the most promising cold cathode material for field emission devices, due to several important advantages. Investigations involving field emission from CVD diamond have largely focused on fabricating diamond

\footnotetext{
* Corresponding author. Tel.: +886-3-5731898; fax: +886-35723811.

E-mail address: cfchen@cc.nctu.edu.tw (C. Chen).
}

films, while several others have fabricated diamond-clad silicon FEAs [2], or researched electron emission from CVD-grown diamond films [3] and silicon tips with diamond particles at their apex end-regions [4]. Furthermore, several other investigations have fabricated pyramidal-shaped diamond field emitters [5]. However, this process is too long, thereby decreasing the quality of, and ultimately weakening, the diamond films, to the extent that it is difficult to carry out treatment by the last process. Hence, in this paper, we report the fabrication of miniature-sized triode diamond FEAs by a simple process.

This work investigates the emission properties of diamond or diamond-like carbon (DLC) films, because certain crystallographic diamond orientations exhibit a negative electron affinity (NEA). This implies that the bottom of the conduction band of the diamond structure lies above the vacuum level, and, therefore, there is no potential barrier for electron emission from the 
conduction band. The electron emission current density is a function of, not only the barrier height, but also of the electron supply function, which subsequently depends on the electron concentration in the conduction band. The conduction band must be populated by n-type doping and/or by an injection of carriers from a metal contact to obtain significant electron emission, because of the wide diamond band-gap. Meanwhile, although the diamond electron emission mechanisms remain unclear, numerous investigators have confirmed that diamond, DLC coatings, and blanket films do exhibit a lower effective work function than Mo or Si.

The electric field, turn-on voltage, and emission current of FEDs depend on the gate-tip spacing, gate aperture diameter, and emitter morphologies. This work compares the influence of field emission characterization between two types of non-doped and P-doped diamond emitters: dendrite-like and nanotube-like diamond FEAs with a $4-\mu \mathrm{m}$ gate aperture.

\section{Experimental}

The fabrication process for this MIS diode and the diamond deposition procedure have been presented previously [6]. First, we designed and fabricated the MIS diode structure using semiconductor process technologies. The starting substrates were mirror-polished n-type, (100)-oriented wafers with a resistivity of $4.5 \sim$ $5.5 \Omega \mathrm{cm}$. After fabricating the MIS diode, specimens were placed in a bias-assisted microwave-plasma chemical vapor deposition (BAMPCVD) system to deposit diamond with various deposition parameters. The reactive gases used in deposition were a conventional mixture of $\mathrm{CH}_{4}-\mathrm{H}_{2}$ with trimethylphosphite $\left[\mathrm{P}\left(\mathrm{OCH}_{3}\right)\right]$ as the doping source. All the diamond deposition experiments used two-step depositions. The first step determines the nucleation process: the flow rates of $\mathrm{CH}_{4} / \mathrm{H}_{2}$ and deposition time remain constant at $10 / 200 \mathrm{sccm}$ and $30 \mathrm{~min}$, respectively. However the second step is the growth process, and total deposition normally lasts for $60 \mathrm{~min}$. During the deposition process, a negative bias voltage of $130 \mathrm{~V}$ was applied to the specimens. Table 1 lists the experimental conditions.

Scanning electron microscopy (SEM) was used to observe the morphology of triode-shaped diamond FEAs with a gated structure; micro-Raman spectroscopy was used to identify the quality of the two different diamond emitters; and an $I-V$ measuring system was used for their electrical characterization. The field emission properties of the triode diamond FEAs were measured using a triode technique. An anode plate (ITO glass) was placed at $100 \mu \mathrm{m}$ above the Pt gate and was biased to $+800 \mathrm{~V}$. The anode current $\left(I_{\mathrm{A}}\right)$ was then measured as a function of the gate-to-cathode
Table 1

Diamond deposition conditions ${ }^{\mathrm{a}}$

\begin{tabular}{lll}
\hline Sample & \multicolumn{2}{l}{$\mathrm{CH}_{4} / \mathrm{H}_{2}+\mathrm{P}\left(\mathrm{OCH}_{3}\right)_{3}(\mathrm{sccm})$} \\
\cline { 2 - 3 } & First step flow rate & Second step flow rate \\
\hline A & $10 / 200$ & $5 / 100$ \\
B & $10 / 200$ & $1 / 200$ \\
C & $10 / 200$ & $2 / 200$ \\
D & $10 / 200+2$ & $2 / 200+2$ \\
E & $10 / 200+1$ & $2 / 200+1$ \\
F & $10 / 200+0.5$ & $2 / 200+0.5$ \\
\hline
\end{tabular}

${ }^{\mathrm{a}}$ First step deposition time, $30 \mathrm{~min}$; second step deposition time, $30 \mathrm{~min}$; bias voltage, $-130 \mathrm{~V}$; MW power, $300 \mathrm{~W}$; total pressure, 15 torr; substrate temperature, $\sim 660^{\circ} \mathrm{C}$.

bias voltage in a vacuum of $1 \times 10^{-6}$ torr. The gate-tocathode voltage $\left(V_{\mathrm{gc}}\right)$ was biased from 0 to $120 \mathrm{~V}$. During testing, the device was in a common emitter configuration, with both the anode and gate being positive potentials to turn the device on.

\section{Results and discussion}

Fig. 1 presents a SEM photograph of the non-doped diamond emitter, while Table 1 list the deposition parameters. Non-doped diamond emitters have several branches on their lateral or top directions. Although more branches are raised as the methane concentration increases, the shape of the diamond does not significantly change with different methane concentrations. Excessive methane concentration causes either the bare silicon substrate to remain on the hole, or the surface of the Pt-gate to be full of diamond. This methane excess affects the Pt-gated electrical properties during the $I-V$ experiment. Indeed, diamond may replace the $\mathrm{Pt}$ to form diamond-gated FEAs in the worst-case scenario. This phenomenon will taint the $I-V$ measurement because the field emission current may be inexact, due to influence from both the diamond emitters and the diamond-gated surface.

Fig. 2 displays the SEM photograph of phosphorusdoped diamond emitters where the doping source is trimethylphosphite $\left[\mathrm{P}\left(\mathrm{OCH}_{3}\right)_{3}\right]$. The branches disappear when phosphorus is added, while the shape resembles nanotube-like diamond. We suspect that phosphorus doping cannot form dendrite-like diamond because of the following reasons. The lower growth rate resulting from higher amounts of trimethylphosphite may be related to the reduction in the $\mathrm{CH}_{4}$ flow rate and the diamond growth precursors in the $\mathrm{H}_{2}-\mathrm{CH}_{4}$ feed gas. Trimethylphosphite is a $\mathrm{CH}_{3}$-rich compound that decomposes in plasma and produces an equal quantity of $\mathrm{CH}_{3}$ radicals to balance the carbon source in the gas phase. The lower growth rates of heavily or slightly doped films are most likely due to the oxygen content 
(a) First step: $\mathrm{CH}_{4} / \mathrm{H}_{2}=10 / 200 \mathrm{sccm}$, Second step: $\mathrm{CH}_{4} / \mathrm{H}_{2}=5 / 200 \mathrm{sccm}$

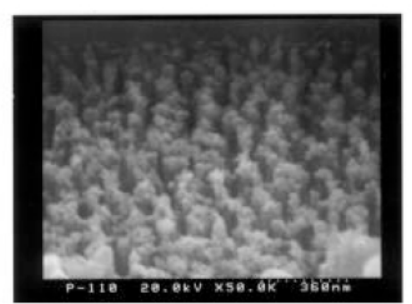

(b) First step: $\mathrm{CH}_{4} / \mathrm{H}_{2}=10 / 200$ sccm, Second step: $\mathrm{CH}_{4} / \mathrm{H}_{2}=1 / 200 \mathrm{sccm}$

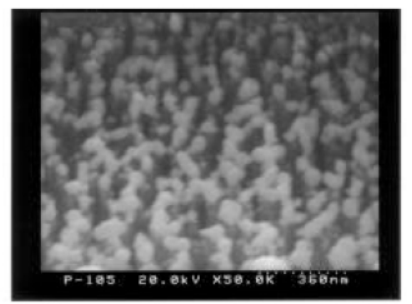

(c) First step: $\mathrm{CH}_{4} / \mathrm{H}_{2}=10 / 200 \mathrm{sccm}$, Second step: $\mathrm{CH}_{4} / \mathrm{H}_{2}=2 / 200 \mathrm{sccm}$

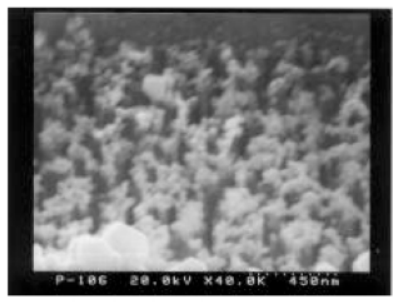

Fig. 1. SEM photographs of non-doped diamond samples grown at different methane concentrations in the second step: (a) sample A; (b) sample B; and (c) sample C.

of trimethylphosphite molecules. There are more oxygen than phosphorus atoms in the doping source, which may be lead to etching of non-diamond carbon from the growing surface, resulting in a lower growth rate. The same effects may also occur when adding $\mathrm{O}_{2}$ to the $\mathrm{CH}_{4}-\mathrm{CO}_{2}$ gas mixture [7].

Many investigations have confirmed that phosphorus will lower the diamond growth rate in $\mathrm{H}_{2} / \mathrm{CH}_{4}$ mixtures [7]. The shape of the emitters resembles a diamond tip with many tiny tips around it when the dopant concentration is $2 \mathrm{sccm}$. The average height and density are smaller than those of non-doped diamond emitters. Decreasing the concentration to 1 and 0.5 sccm enhances both the intensity and average height of the diamond emitters, which have some invisible branches on their bodies at $0.5 \mathrm{sccm}$. Lowering the phosphorus concentration transforms the shape from nanotube-like to dendrite-like diamond, and increases the amount of visible emitters.

The SEM images indicate that diamond films can be uniformly grown within the Pt-gated FEAs on the Pt-gated surfaces that do not nucleate diamond. In addition, the O-ring phenomenon [8] does not appear around the Pt-gated pattern. These results are attributed to the local biased effect [9] and selective area deposition diamond effect for the following two reasons. First, it is easier to grow synthetic diamond on silicon than a Pt-gated layer, because $\mathrm{Pt}$ is a highly chemically inert material that does not nucleate diamond on an unscratched surface. Second, although the bias current concentrated on the silicon substrate surface occurs because the n-type silicon conducts electrons, electrons cannot pass through silicon dioxide with a Pt-gated layer. Therefore, the local diamond deposition biased effects are within the silicon substrate, while the O-ring phenomenon does not appear around the Pt-gated pattern.

Fig. 3 depicts the micro-Raman spectrum of the non-doped diamond on the Pt-gated FEAs, which confirms various methane concentrations during diamond emitter growth. Although the 5\%-methane series (samples A, B and C) displayed a $1580 \mathrm{~cm}^{-1}$ shoulder, the G-band shrank and was not very clear. Characterizing these diamond emitters is often difficult, because they contain excessive graphite crystallization. Indeed, un-

(a) $\mathrm{P}\left(\mathrm{OCH}_{3}\right)_{3}=2 \mathrm{sccm}$
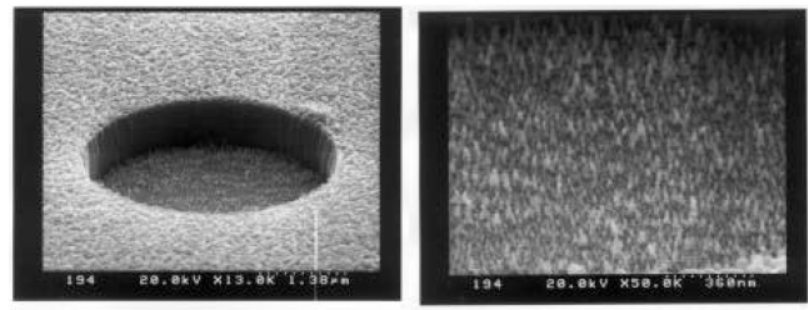

(b) $\mathrm{P}\left(\mathrm{OCH}_{3}\right)_{3}=1 \mathrm{sccm}$

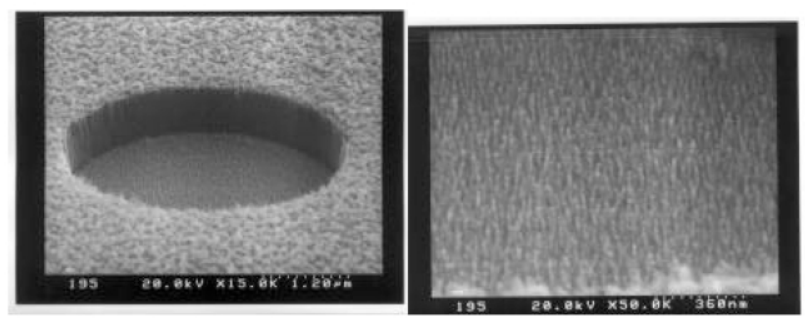

(c) $\mathrm{P}\left(\mathrm{OCH}_{3}\right)_{3}=0.5 \mathrm{sccm}$
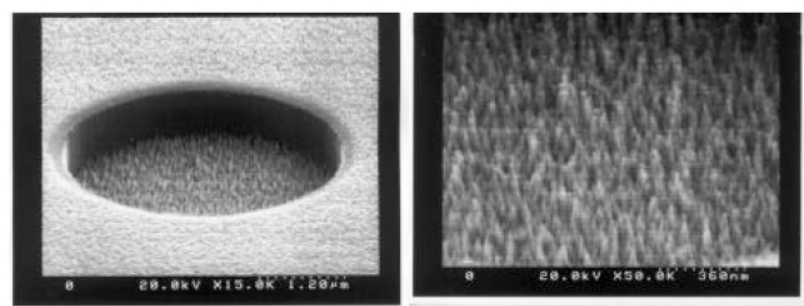

Fig. 2. SEM photographs of P-doped diamond samples with different concentrations of $\mathrm{P}\left(\mathrm{OCH}_{3}\right)_{3}$ dopant: (a) sample D; (b) sample E; and (c) sample F. 


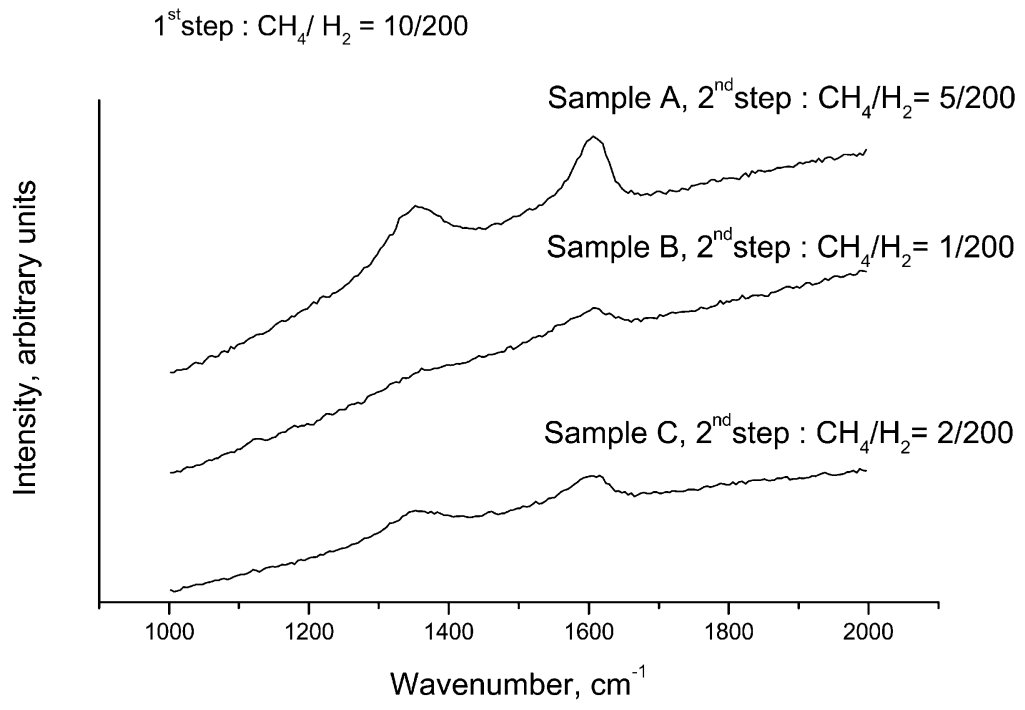

Fig. 3. Raman spectrum of non-doped diamond samples A, B and C grown at different methane concentrations in the second step.

stable methane concentrations during the experimental process will create variations in the two distinct peaks.

Fig. 4 reveals that decreasing phosphorus content causes the G-band to broaden. On the other hand, the peak at $1580 \mathrm{~cm}^{-1}$ shifts to $1606 \mathrm{~cm}^{-1}$, indicating that graphite may reside between the diamond grain boundaries. This Raman shift occurs because of internal stress in the diamond film. This stress could be attributed to the following reasons: (i) the lattice mismatch between diamond and phosphorus during growth; and (ii) the thermal expansion coefficients of diamond and the substrate, as well as the microcrystalline nature of the diamond. A sharp peak appearing at 1332.5 $\mathrm{cm}^{-1}$ would be the main characteristic of well-crystallized diamond. But strictly speaking, the nano-doped and P-doped diamonds in our work are diamond-like carbon (DLC). Thus, they only have peaks at approximately $1350 \mathrm{~cm}^{-1}$ in the region of the characteristic sharp peak (at $1332.5 \mathrm{~cm}^{-1}$ ).

Comparing Raman spectra of the non-doped and P-doped diamond reveals that the quantity of graphite increased and the diamond qualities decreased on doping, since the intensity of the broad peak centered at $1580 \mathrm{~cm}^{-1}$ increased with trimethylphosphite concentration. This phenomenon is clearer when phosphorus doping with trimethylphosphite, as the methyl radical $\left(\mathrm{CH}_{3}^{\bullet}\right)$ concentration increases with that of the dopant, as it is a $\mathrm{CH}_{3}$-rich compound. Thus, the etching rate declined, while the quantity of amorphous carbon or graphite increased in the resultant films. The Raman shift can be accounted for by the lattice strain caused by phosphorus doping.

In this work, there are significant differences between non-doped and P-doped diamond. Fig. 5 shows the field emission current $\left(I_{\mathrm{a}}\right)$ vs. the gate voltage of non-doped dendrite-like P-doped nanotube-like diamond. Phos- phorus doping can enhance the electrical properties by increasing the emission current, since the field emission current $\left(I_{\mathrm{a}}\right)$ of non-doped and P-doped emitters is $4 \mu \mathrm{A}\left(\right.$ at $\left.V_{\mathrm{gc}}=45 \mathrm{~V}\right)$ and $322 \mu \mathrm{A}\left(\right.$ at $\left.V_{\mathrm{gc}}=120 \mathrm{~V}\right)$, respectively. The emission current of P-doped diamond is 80 -fold larger than that of non-doped diamond. The experimental results confirm that P-doped diamond has better field emission characteristics than non-doped material, because the former has a higher electron conductivity and defect density. A display requires 0.1 $\mathrm{mA} \mathrm{cm}{ }^{-2}$ to excite the phosphorus pixel. Thus, a field emission current density of approximately 13 and 1026 $\mathrm{mA} \mathrm{cm}{ }^{-2}$ must be obtained for non-doped and P-doped diamond, respectively.

There is a saturated current at $V_{\mathrm{gc}}=45$ and $120 \mathrm{~V}$ in non-doped and P-doped diamond emitter arrays, respectively. This saturation can be accounted for in two ways. First, by the tip effect of the diode structure, where the far distance between the Pt gate and the diamond tip depends on the size of diamond tip and the aperture depth. Thereby, part of the field emission current flows into the $\mathrm{Pt}$ gate and the anode current is reduced. The other reason is the dielectric layer $\left(\mathrm{SiO}_{2}\right)$ in the developed pattern may be broken, which will create a leakage current when a high voltage is applied.

The electron-emitting characteristics of these two types of diamond were further evaluated using a Fowler-Nordheim plot. The threshold voltage $\left(V_{\text {th }}\right)$ is defined as the intersection of the slope of the F-N plots. The threshold voltage $\left(V_{\mathrm{th}}\right)$ significantly changes for non-doped and P-doped diamond emitter arrays: 15 and $5 \mathrm{~V}$, respectively.

Experimental results indicate that P-doped FEAs have better field emission characteristics than nondoped ones. Solid-state physics proves that phosphorus doping will instigate an energy bend diagram change by 
(a) $\mathrm{P}\left(\mathrm{OCH}_{3}\right)_{3}=2 \mathrm{sccm}$

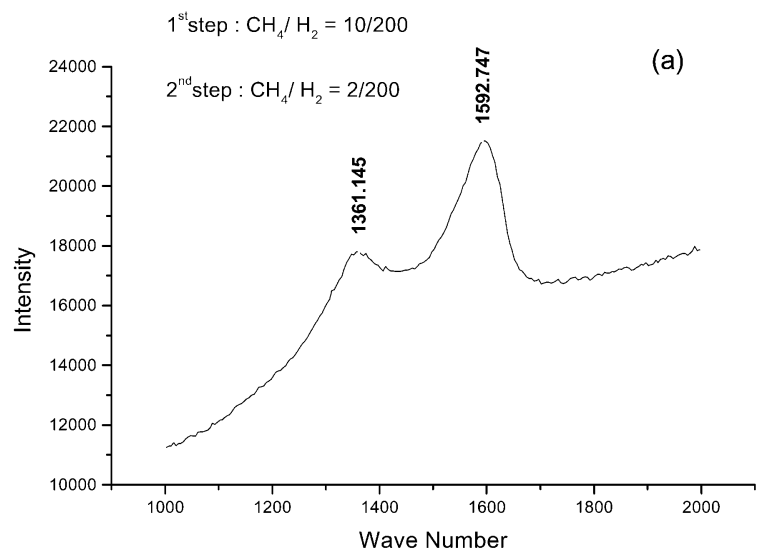

(b) $\mathrm{P}\left(\mathrm{OCH}_{3}\right)_{3}=1 \mathrm{sccm}$

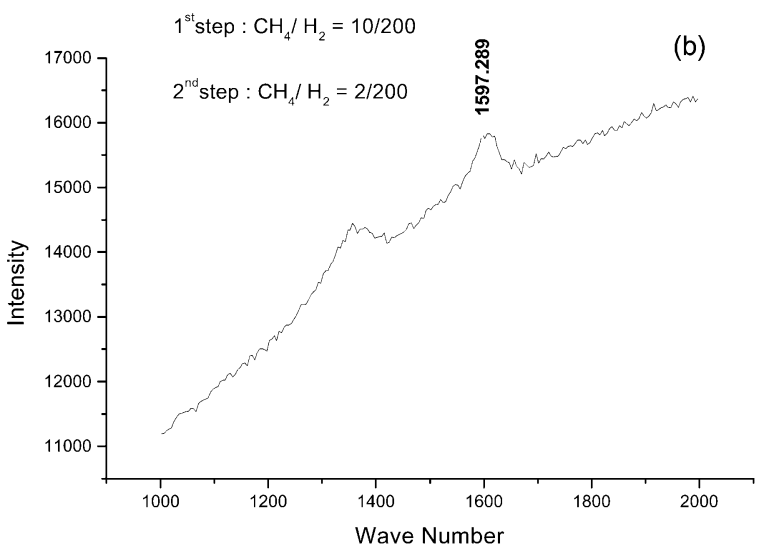

(c) $\mathrm{P}\left(\mathrm{OCH}_{3}\right)_{3}=0.5 \mathrm{sccm}$

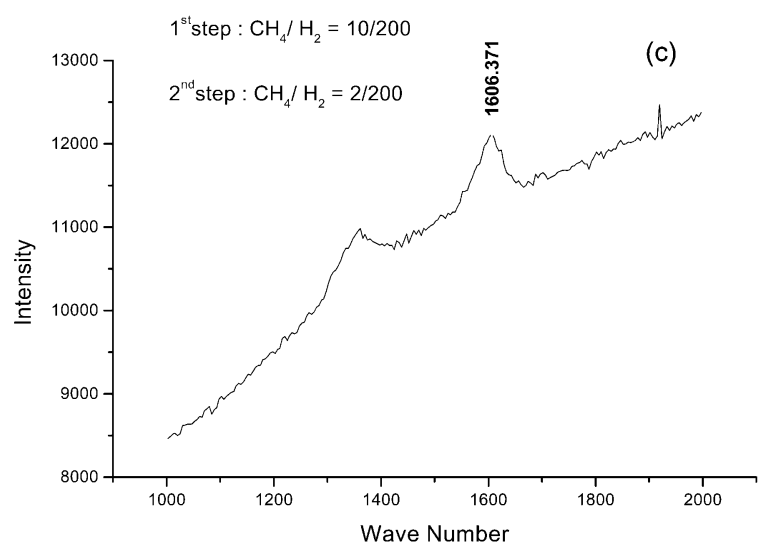

Fig. 4. Raman spectra of P-doped diamond samples with different concentrations of $\mathrm{P}\left(\mathrm{OCH}_{3}\right)_{3}$ dopant: (a) sample D; (b) sample E; and (c) sample F.
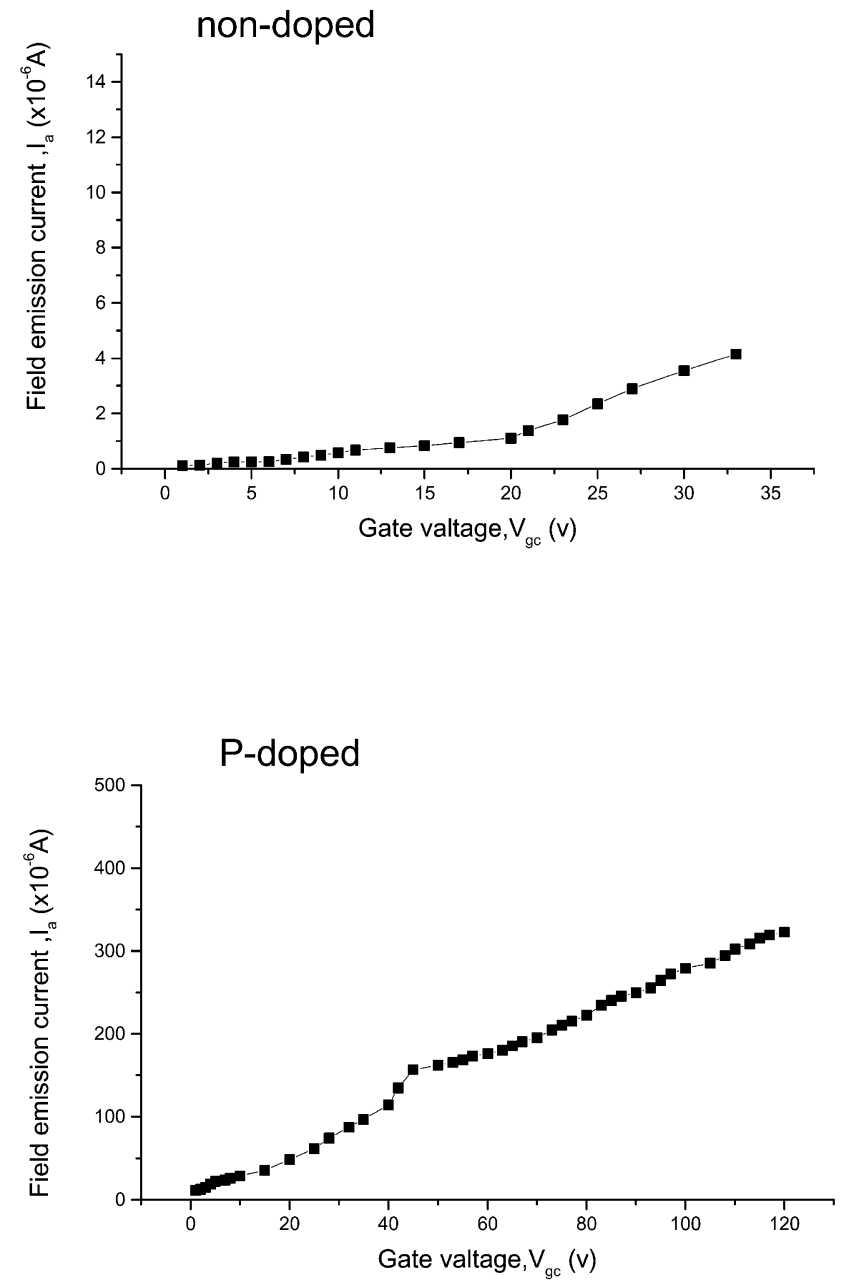

Fig. 5. The field emission current versus the gate voltage of nondoped dendrite-like diamond and P-doped nanotube-like diamond.

generating a donor level that provides more electrons for the material. This increases the total conductivity and the net flux. P-doped material has many defects and provides its electrons with a greater opportunity to be extracted, because ionized donors form the spacecharge layer [10].

\section{Conclusions}

In this work, we demonstrate the feasibility of a new fabrication process for triode diamond FEAs, which contain a small gate aperture and a shallow depth of field cells. Doping with phosphorus can enhance the electrical characteristic, by reducing the turn-on voltage and enhancing the emission current density. The turn-on voltage for non-doped and P-doped emitters is 15 and $5 \mathrm{~V}$, respectively. The field emission current $\left(I_{\mathrm{a}}\right)$ of non-doped and P-doped emitters is $4 \mu \mathrm{A}$ (at $V_{\mathrm{gc}}=45$ $\mathrm{V})$ and $322 \mu \mathrm{A}\left(\right.$ at $V_{\mathrm{gc}}=120 \mathrm{~V}$ ). The emission current of P-doped emitters is approximately 80 -fold larger 
than that of non-doped diamond emitters. Selective area deposition (SAD) of diamond on the silicon substrates was successfully achieved by using a Pt-gated layer as a nucleation inhibitor. Two types of emitters were formed: non-doped dendrite-like and P-doped nanotube-like diamond FEAs.

\section{Acknowledgements}

The authors would like to thank the National Science Council of the Republic of China for financially supporting this research under Contract No. NSC892216-E-009-007.

\section{References}

[1] J.S. Lee, K.S. Liu, I.N. Lin, Appl. Phys. Lett. 71(4) (1997) 554.

[2] N.J. She, A Study of Application and Characterization of
Diamond Thin Films on Field Emitter Arrays, Chiao Tung University, 1996 (Masters thesis).

[3] W.H. Huang, Study of Selective Growth of Diamond Film for Field Emitter Arrays, Chiao Tung University, 1996 (Masters thesis).

[4] E.I. Givargizov, V.V. Zhirnov, A.N. Stepanova, E.V. Rakova, A.N. Kiselev, P.S. Plekhanov, Microstructure and field emission of diamond particles on silicon tips, Appl. Surf. Sci. 87/88 (1995) 24.

[5] E.S. Baik, D.R. Jeon, Y.J. Baik, Diamond. Relat. Mater. 8 (1999) 89-93.

[6] C.F. Chen, H.C. Wang, H.C. Hsieh, Jpn. J. Appl. Phys. 39 (2000) 1880-1884.

[7] C.F. Chen, T.M. Hang, S.H. Cheng, J. Appl. Phys. 74 (1993) 4483.

[8] D.R. Wang, Fabrication and Characterization of the Sub-Micro Cone-Shaped Diamond Field Emitter Arrays, Chiao Tung University, 1997 (Masters thesis).

[9] R. Stochel, K. Janischowsky, S. Rohmfeld, J. Ristein, M. Hundhausem, L. Ley, Diamond Relat. Mater. 5 (1996) 321.

[10] K. Kuriyama, C. Kimura, S. Koizumi, M. Kamo, T. Sugino, J. Vac. Technol. B 17 (2) (1999) 723. 